\title{
Paraganglioma gangliocítico en paciente varón de 24 años: causa infrecuente de sangrado gastrointestinal
}

\author{
Gangliocytic paraganglioma in a 24-year-old male patient: an infrequent cause of \\ gastrointestinal bleeding
}

\author{
Diego Téllez-Beltrán ${ }^{1}$, Alejandro González-Muñoz ${ }^{2 *}$, Fabio E. Pinzón ${ }^{1}$, Andrés Lanza ${ }^{2}$ y Manuela Pineda ${ }^{1}$ \\ ${ }^{1}$ Servicio de Cirugía General, Hospital Universitario San Ignacio; ${ }^{2}$ Servicio de Cirugía General, Pontificia Universidad Javeriana. Bogotá, Colombia
}

\begin{abstract}
Resumen
Los paragangliomas gangliocíticos son tumores neuroendocrinos infrecuentes del sistema gastrointestinal, generalmente de curso benigno y en la gran mayoría de los casos asintomáticos. Presentamos el caso de un paciente joven, previamente sano, quien consulta por dolor abdominal y hemorragia de vías digestivas altas. En la endoscopia de vías digestivas altas se evidencia una lesión pediculada en la segunda porción del duodeno. Se decide realizar laparotomía mediana y duodenectomía con resección del tumor duodenal pediculado. Los resultados del estudio histológico e inmunohistoquímico fueron compatibles con paraganglioma gangliocítico duodenal. Posterior al procedimiento presenta adecuada recuperación, sin evidencia de recidiva en el seguimiento.
\end{abstract}

Palabras clave: Duodeno. Paraganglioma. Sangrado digestivo.

\begin{abstract}
Gangliocytic paragangliomas are infrequent neuroendocrine tumors, usually of benign course and in the vast majority without any symptoms reported. We present the case of a young, previously healthy patient, who consults for abdominal pain and upper gastrointestinal bleeding. Through an endoscopy of the upper digestive tract, a pedicle lesion is evidenced in the second portion of the duodenum. We decided to perform a medium laparotomy and duodenectomy with resection of pedicle duodenal tumor. The results of the histological and immunohistochemical study were compatible with a duodenal gangliocytic paraganglioma. After the procedure, the patient presents adequate recovery without evidence of recurrence in the follow-up.
\end{abstract}

Key words: Duodenum. Paraganglioma. Gastrointestinal bleeding.

\section{Introducción}

Los tumores neuroendocrinos en el tracto gastrointestinal suelen caracterizarse por tener un curso benigno, en comparación a los adenocarcinomas en dicha región ${ }^{1-3}$. Este grupo de neoplasias representan cerca del $4 \%$ de los carcinomas gastrointestinales ${ }^{2}$, correspondiendo a una categoría infrecuente de tumores. 
Dentro de esta clasificación se encuentran los paragangliomas gangliocíticos (PG), tumores derivados de la cresta neural ${ }^{4}$, descritos por primera vez en 1957 como ganglioneuromas ${ }^{5}$, y en 1971 denominados PG por las tres variantes celulares que conforman estos tumores: células epitelioides, ahusadas y gangliocíticas $^{1-3,5,6}$. Esta variante corresponde al 6-9\% de los tumores neuroendocrinos duodenales, siendo más frecuentes los gastrinomas y los somatostatinomas ${ }^{2,7}$.

El rango de edad en que más frecuentemente se presenta el PG es bastante amplio, identificando casos en pacientes entre 15 y 84 años, sobre todo en hombres mayores de 50 años $^{1,8}$. La localización donde más se presentan los $P G$ es la segunda porción del duodeno $0^{1,3,8-10}$, y con menor frecuencia en el esófago, el mediastino, el timo, los pulmones y los ovarios $^{8}$. En la mayoría de los casos el comportamiento del PG ha mostrado ser benigno, existiendo pocos casos documentados de compromiso de la totalidad de la pared duodenal, metástasis ganglionares o locales, o recurrencia $a^{4,6,9-12}$.

La clínica es esencial para el diagnóstico, lo cual se encuentra correlacionado con la progresión de la enfermedad ${ }^{2}$, hasta asociarse a sus principales manifestaciones, dentro de las cuales se encuentran sangrado gastrointestinal, dolor abdominal e ictericia obstructiva ${ }^{7,8}$. Su identificación histopatológica se logra gracias a la presencia de marcadores neuroendocrinos fundamentales para su diagnóstico, como la cromogranina A, la sinaptofisina, el Leu7 y la enolasa neuronal específica ${ }^{1-3,13}$.

El único tratamiento definitivo es el quirúrgico, el cual se puede realizar de manera endoscópica, laparoscópica o cirugía abierta ${ }^{12,14,15}$. A continuación presentamos el caso de un paciente de 24 años, quien debutó con una hemorragia de vías digestivas secundaria a la presencia de un PG.

\section{Caso clínico}

Varón de 24 años sin antecedentes médicos, que consulta al servicio de urgencias por un cuadro clínico de 15 días, consistente en dolor abdominal tipo cólico, de intensidad inicialmente leve, pero progresiva, no irradiado, asociado a sensación de plenitud posprandial, pirosis y dispepsia; posteriormente el paciente refiere presencia de deposiciones melénicas y un episodio aislado de hematoquecia.

Se decide hospitalizar al paciente para realización de estudios, en los que se evidencia anemia normocítica, normocrómica, sin requerimiento de transfusión

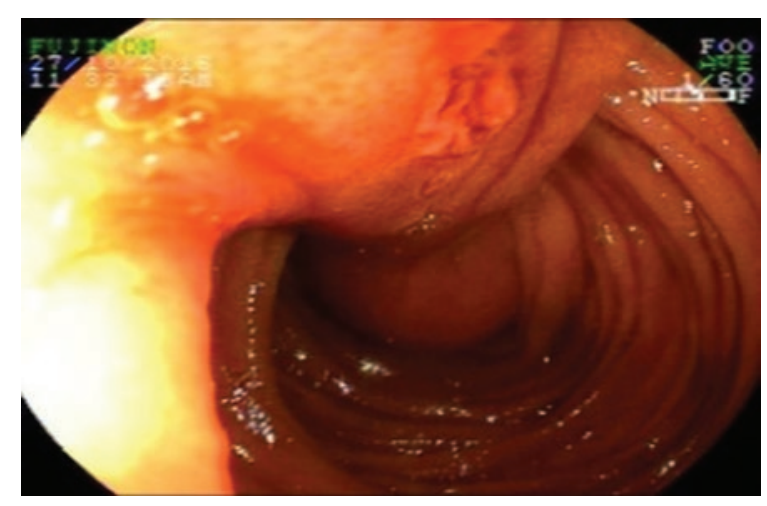

Figura 1. Endoscopia de vías digestivas altas, en la cual se evidencia, en la segunda porción del duodeno, una lesión de aspecto subepitelial, pediculada, con ulceración central, sin compromiso de la papila duodenal.

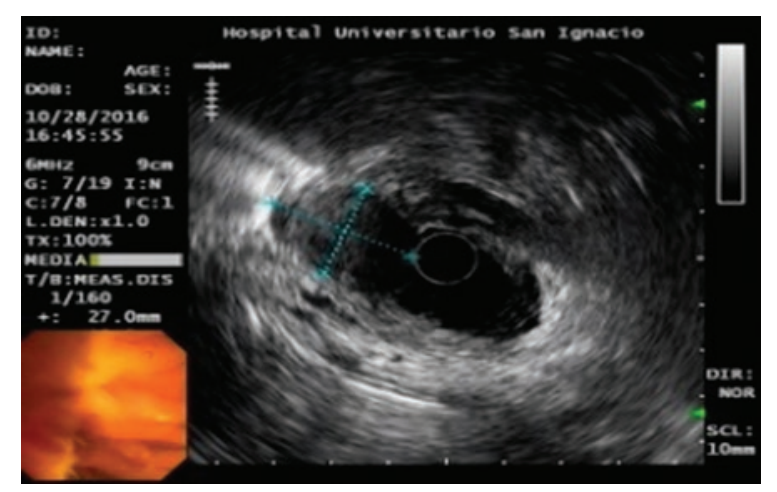

Figura 2. Ecoendoscopia, con evidencia de lesión hipoecoica, homogénea, de $29 \times 19 \mathrm{~mm}$, de bordes bien definidos, en el duodeno.

de hemoderivados, por presencia de hemorragia de vías digestivas altas. Se decide realizar esofagogastroduodenoscopia (Fig. 1), en la cual se identifica, en la segunda porción del duodeno, una lesión pediculada con ulceración central, sin compromiso de la papila duodenal, la cual se encuentra más proximal a la lesión descrita, aproximadamente a $1 \mathrm{~cm}$. Adicionalmente se realiza ecoendoscopia (Fig. 2), que reporta una lesión hipoecoica, homogénea, de aproximadamente $29 \times 19 \mathrm{~mm}$, de aspecto subepitelial, en la segunda porción del duodeno, pendulante, con pedículo ancho y erosiones en su superficie, con sospecha diagnóstica de tumor del estroma gastrointestinal.

Se decide llevar el paciente a la sala de cirugía, donde por medio de laparotomía mediana se accede a la cavidad abdominal y se identifican el píloro y la segunda porción del duodeno; a través de una duodenotomía se evidencia la masa tumoral de aspecto estromal, de aproximadamente $3 \times 2 \mathrm{~cm}$, con 


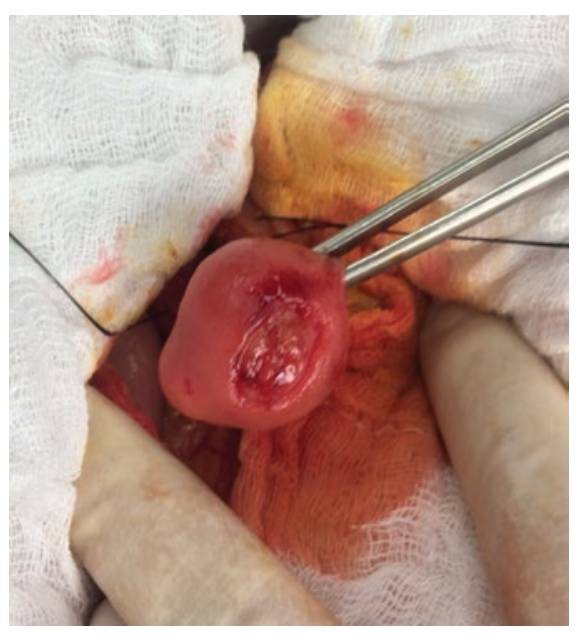

Figura 3. Masa polipoide expuesta a través de duodenotomía, con presencia de ulceración central. De manera intraoperatoria no se identifica sangrado activo.

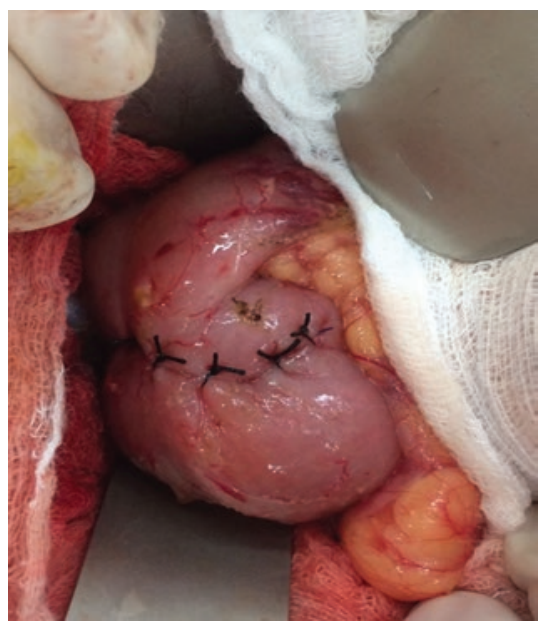

Figura 4. Duodenorrafia cerrada con seda 0000, sin evidencia de fugas.

ulceración central. Se denota lesión dependiente de la submucosa, en la pared medial del duodeno, a $1,5 \mathrm{~cm}$ de la papila (Fig. 3).

Se realiza resección total de la lesión con éxito y posteriormente se lleva a cabo una duodenorrafia (Fig. 4), con muestra patológica consistente en masa tumoral de aspecto estromal, ulcerada, de aproximadamente $3 \times 2 \mathrm{~cm}$ (Fig. 5), y se cierra la pared abdominal. No se reportan complicaciones, sangrado ni otras eventualidades durante el procedimiento.

Durante el posoperatorio, el paciente presenta una evolución clínica favorable, iniciando de forma temprana la vía oral y la marcha, con posterior recuperación completa, por lo cual se decide dar egreso hospitalario.

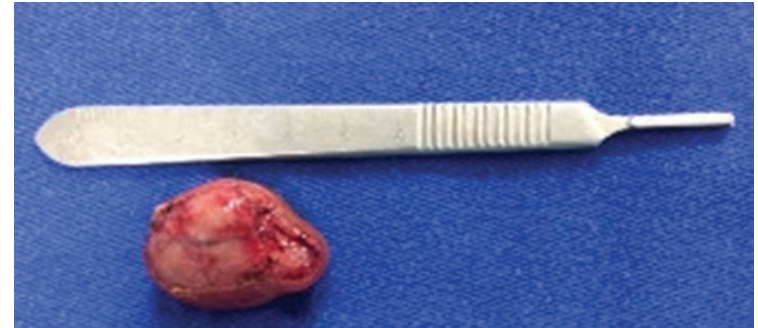

Figura 5. Masa tumoral de aspecto estromal, ulcerada, de aproximadamente $3 \times 2 \mathrm{~cm}$, de localización submucosa, dependiente de la pared medial del duodeno, a 1,5 cm de la papila duodenal, mayor en sentido caudal.

El estudio histopatológico reporta presencia de células epitelioides con núcleos fusiformes y células ganglionares, con estudio inmunohistoquímico positivo para reactividad con enolasa neuronal específica, sinaptofisina y proteína S-100, realizando diagnóstico de PG. En la muestra se evidencian márgenes libres de lesión (Fig. 6). El paciente sigue control ambulatorio y a los 6 meses de seguimiento presenta una adecuada evolución clínica, sin complicaciones tardías.

\section{Discusión}

De acuerdo con la Organización Mundial de la Salud (OMS), el PG se clasifica como un tumor neuroendocrino de grado 1 de bajo grado, por su reducida actividad proliferativa, con una sobrevida a 5 años del $80-90 \%{ }^{3}$. Su origen aún continúa siendo desconocido; algunas teorías apuntan que corresponde a un hamartoma desarrollado de tejidos embrionarios pancreáticos $^{8,16}$ mientras que otra plantea su procedencia desde los ganglios de origen neuroectodérmico y de células de Schwann ${ }^{5}$.

Okubo, et all.,8 realizaron una caracterización epidemiológica de los casos de PG disponibles en la literatura, identificando cerca de 192 casos reportados, con un promedio de edad de 52,3 años y una proporción hombre:mujer de 114:76. Respecto al tamaño de la lesión, identificaron un diámetro de entre 5,5 y $100 \mathrm{~mm}$, y su localización más frecuente fue el duodeno $(90,2 \%$ de los casos), seguido de la médula espinal, el sistema respiratorio, el yeyuno, el esófago y, con menos frecuencia, el estómago, el apéndice y el mediastino posterior.

Durante el diagnóstico endoscópico se identifica a menudo como la presencia de una masa polipoide ${ }^{2}$, lesión que puede caracterizarse en la tomografía computarizada (TC) como un defecto intraluminal a nivel del duodeno en caso de encontrarse en esta 


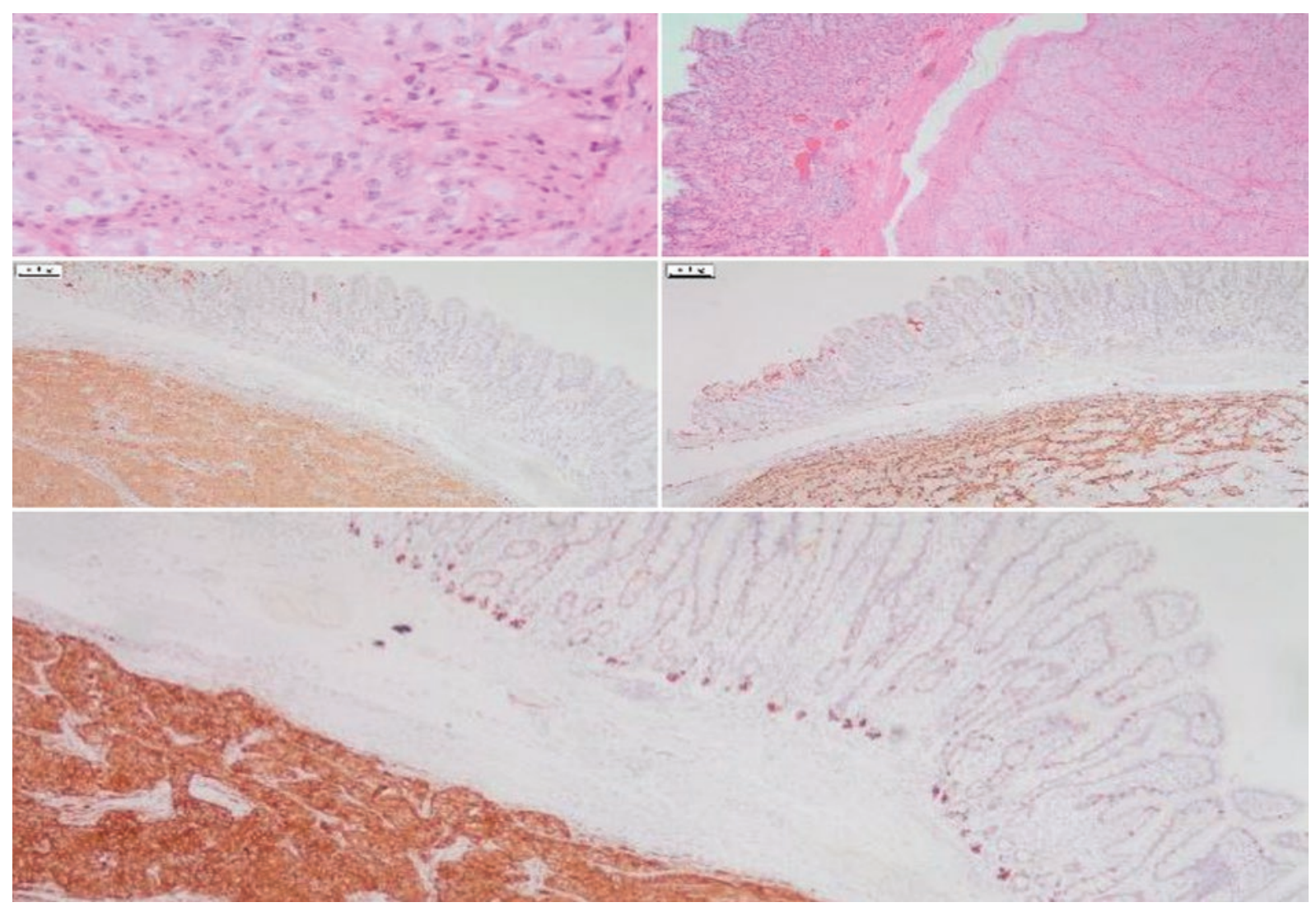

Figura 6. Estudio histológico de muestra patológica. A-B: presencia de células epitelioides con núcleos fusiformes y células ganglionares, visible en placas que corresponde a coloración básica con hematoxilina eosina. C: estudio de inmunohistoquímica mostrando positividad para enolasa neuronal especifica. D: proteína S-100. E: sinaptofisina.

localización ${ }^{17,18}$. También es posible usar la ultrasonografía, con menor rendimiento respecto a las pruebas anteriores, aunque como estudio inicial, por su costo y facilidad de implementación, puede emplearse para una orientación diagnóstica inicial ${ }^{17}$.

El diagnóstico histopatológico con biopsia previa a la intervención quirúrgica parece tener una pobre efectividad ${ }^{6,8}$. Boeriu, et al. ${ }^{2}$ lo atribuyen al compromiso que tiene el PG sobre la submucosa, mostrando un rendimiento cercano al $11,4 \%$.

Respecto a la clínica, el sangrado gastrointestinal se encuentra presente en el $45,1 \%$ de los pacientes, seguido del dolor abdominal $(42,8 \%)$, la anemia $(14,5 \%)$ y la obstrucción de vías biliares $(4,6 \%)^{7,8}$.

Barret, et al..$^{11}$ presentan un algoritmo terapéutico basado en el tamaño de la lesión y en la presencia o no de compromiso linfático. Establecen que los tumores < $2 \mathrm{~cm}$, sin evidencia de compromiso linfático por TC, pueden ser tratados mediante endoscopia, ampulectomía o tumorectomía transduodenal laparoscópica con duodenoscopia preoperatoria, mientras que en los tumores $>2 \mathrm{~cm}$, con sospecha de metástasis por sus características histológicas (infiltración de márgenes en la resección local o alta actividad mitótica), es preferible la realización de una pancreatoduodenectomía con vaciamiento ganglionar. Witzigmann, et al. ${ }^{9}$, basándose en su experiencia en el manejo de PG, recomiendan la realización de vaciamiento ganglionar en caso de tumores $>2 \mathrm{~cm}$ y lesiones con pleomorfismos y con identificación de mitosis. Este último procedimiento se indica adicionalmente cuando existe compromiso de la papila y de la vía biliar, con presencia de ictericia obstructiva ${ }^{19}$. En nuestro caso se decidió realizar un manejo abierto por el tamaño de la lesión, ya que cabía la posibilidad de requerir una resección más amplia o un vaciamiento ganglionar; sin embargo, no identificamos lesiones adicionales ni compromiso ganglionar.

El manejo laparoscópico no parece tener muchas diferencias respecto al manejo abierto, por lo cual se deja la ampulectomía laparoscópica como un tratamiento electivo en este tipo de tumores ${ }^{15}$. Respecto al manejo endoscópico, actualmente se encuentra limitado a lesiones sin compromiso transmural extenso ${ }^{20}$. 
Existen muy pocos reportes en la literatura de pacientes con PG con compromiso metastásico, el cual se ha asociado a una invasión intramural profunda ${ }^{1,12,13,21}$, caracterizando un compromiso metastásico nodular en el 5-7\% de los $\operatorname{casos}^{6}$. Hasta el momento no hay claridad respecto al uso de irradiación o de quimioterapia posterior a la intervención quirúrgica en los pacientes con PG ${ }^{11}$.

Se ha buscado identificar diferentes factores pronósticos del PG, dentro de los cuales se ha intentado implementar el uso de marcadores tumorales, como bcl-2, p53 y KI-67, los cuales muestran tener afinidad por ciertos tumores neuroendocrinos, pero hasta el momento no se ha probado su reactividad con el $\mathrm{PG}^{8}$. Sin embargo, según la OMS, la expresión de KI-67 es el único determinante del pronóstico del $\mathrm{PG}^{22}$. Hasta el momento, el compromiso extenso de la submucosa parecer tener relación con el riesgo de diseminación linfática ${ }^{1,8}$.

El único caso de recurrencia fue reportado por Álvarez-Pertuz, et al. ${ }^{19}$, en un paciente de 37 años, en quien se realizó una resección de un PG por medio de laparotomía, y 2 años después se identificó en su seguimiento, por medio de TC, la presencia de una masa infiltrativa en el yeyuno, clasificada como una recidiva de su primera patología.

\section{Conclusión}

El PG es una neoplasia infrecuente, asintomática en la mayoría de los casos, con un comportamiento benigno según la literatura, hasta presentar manifestaciones como el sangrado digestivo, como ocurrió en nuestro paciente.

El presente caso ilustra un comportamiento benigno de la patología, sin documentación de recidiva hasta el momento, con tratamiento exitoso mediante resección por laparotomía exploratoria.

\section{Agradecimientos}

Agradecemos al Hospital Universitario San Ignacio y a la Pontificia Universidad Javeriana.

\section{Conflicto de intereses}

Los autores no declaran conflictos de intereses.

\section{Responsabilidades éticas}

Protección de personas y animales. Los autores declaran que para esta investigación no se han realizado experimentos en seres humanos ni en animales.
Confidencialidad de los datos. Los autores declaran que han seguido los protocolos de su centro de trabajo sobre la publicación de datos de pacientes.

Derecho a la privacidad y consentimiento informado. Los autores han obtenido el consentimiento informado de los pacientes y/o sujetos referidos en el artículo. Este documento obra en poder del autor de correspondencia.

\section{Bibliografía}

1. Okubo Y, Yokose T, Motohashi O, Miyagi Y, Yoshioka E, Suzuki M, et al. Duodenal rare neuroendocrine tumor: clinicopathological characteristics of patients with gangliocytic paraganglioma. Gastroenterol Res Pract. 2016;2016:5257312.

2. Boeriu A, Dobru D, Georgescu R, Mocan S, Boeriu C. Gangliocytic paraganglioma: a rare cause of gastrointestinal bleeding. J Gastrointest Liver Dis. 2015;24:109-12.

3. Okubo Y, Nemoto T, Wakayama M, Tochigi N, Shinozaki M, Ishiwatari T, et al. Gangliocytic paraganglioma: a multi-institutional retrospective study in Japan. BMC Cancer. 2015;15:269.

4. Narang V, Behl N, Sood N, Puri H. Gangliocytic paraganglioma of duodenum. Case Rep Pathol. 2013;2013:378582.

5. Čečka F, Jon B, Repák R, Kohout A, Šubrt Z, Ferko A. Gangliocytic paraganglioma of the duodenum. Can J Gastroenterol. 2012;26:778-9.

6. Loftus TJ, Kresak JL, Gonzalo DH, Sarosi GAJ, Behrns KE. Duodenal gangliocytic paraganglioma: a case report and literature review. Int $\mathrm{J}$ Surg Case Rep. 2015;8C:5-8.

7. Hoffmann KM, Furukawa M, Jensen RT. Duodenal neuroendocrine tumors: classification, functional syndromes, diagnosis and medical treatment. Best Pract Res Clin Gastroenterol. 2005;19(5 Spec Iss):675-97.

8. Okubo Y, Wakayama M, Nemoto T, Kitahara K, Nakayama H, Shibuya K, et al. Literature survey on epidemiology and pathology of gangliocytic paraganglioma. BMC Cancer. 2011;11:187.

9. Witzigmann H, Loracher C, Geissler F, Wagner T, Tannapfel A, Uhlmann $D$, et al. Neuroendocrine tumours of the duodenum: clinical aspects, pathomorphology and therapy. Langenbeck's Arch Surg. 2002; 386:525-33.

10. Hernandez AG, Lanuza ED-A, Matias AC, Huertas RP, Rodriguez KMG, Perez PG, et al. Large gangliocytic paraganglioma of the duodenum: a rare entity. World J Gastrointest Surg. 2015;7:170-3.

11. Barret M, Rahmi G, Duong van Huyen JP, Landi B, Cellier C, Berger A. Duodenal gangliocytic paraganglioma with lymph node metastasis and an 8-year follow-up: a case report. Eur J Gastroenterol Hepatol. 2012; 24:90-4.

12. Papaconstantinou D, Machairas N, Damaskou V, Zavras N, Kontopoulou C, Machairas A. Duodenal gangliocytic paraganglioma, successfully treated by local surgical excision - a case report. Int J Surg Case Rep. 2017;32:5-8.

13. Vanoli A, La Rosa S, Klersy C, Grillo F, Albarello L, Inzani F, et al. Four neuroendocrine tumor types and neuroendocrine carcinoma of the duodenum: analysis of 203 cases. Neuroendocrinology. 2016;104:112-25.

14. Rafiullah M, Tanimu S, Onitilo AA. Gangliocytic paraganglioma, a rare ampullary tumour treated with open transduodenal resection and sphincteroplasty. BMJ Case Rep. 2014;2014:bcr2013202941.

15. Ahn KS, Han HS, Yoon YS, Cho JY, Khalikulov K. Laparoscopic transduodenal ampullectomy for benign ampullary tumors. J Laparoendosc Adv Surg Tech. 2010;20:59-63.

16. Matsubayashi $H$, Ishiwatari $H$, Matsui $T$, Fujie $S$, Uesaka K, Sugiura T, et al. Gangliocytic paraganglioma of the minor papilla of the duodenum. Intern Med. 2017;56:1029-35.

17. Buetow PC, Levine MS, Buck JL, Pantongrag-Brown L, Emory TS. Duodenal gangliocytic paraganglioma: CT, MR imaging, and US findings. Radiology. 1997;204:745-7.

18. Plaza JA, Vitellas K, Marsh WLJ. Duodenal gangliocytic paraganglioma: a radiological-pathological correlation. Ann Diagn Pathol. 2005;9:143-7.

19. Álvarez-Pertuz $\mathrm{H}$, Chacón-Garita L. Paraganglioma gangliocítico en la tercera porción del duodeno. Acta Med Costarric. 2015;57:44-6.

20. Guerges M, Slama E, Maskoni B, Imlay S, McKany M. Gangliocytic paraganglioma treated with ampullectomy. A case report. Ann Med Surg. 2016;11:16-20.

21. Witkiewicz A, Galler A, Yeo CJ, Gross SD. Gangliocytic paraganglioma: case report and review of the literature. J Gastrointest Surg. 2007;11:1351-4.

22. Tatangelo F, Cantile M, Pelella A, Losito NS, Scognamiglio G, Bianco F, et al. Duodenal gangliocytic paraganglioma, a rare entity among GEPNET: a case report with immunohistochemical and molecular study. Diagn Pathol. 2014;9:54. 